Rev. Inst. Flor. v. 29 n. 2 p. $157-167$ dez. 2017

http://dx.doi.org/10.24278/2178-5031.201729202

ISSN impresso 0103-2674/on-line 2178-5031

\title{
EMERGÊNCIA E CRESCIMENTO DE MUDAS DE Leucaena leucocephala CULTIVADAS EM DIFERENTES SUBSTRATOS ${ }^{1}$
}

\section{EMERGENCY AND SEEDLINGS GROWTH OF Leucaena leucocephala CULTIVATED IN DIFFERENT SUBSTRATES}

\author{
Genilda Canuto AMARAL ${ }^{2,7}$; Adênio Louzeiro AGUIAR JÚNIOR³; \\ Yara Karolynne Lopes ABREU4; ${ }^{4}$ Márkilla Zunete Beckmann CAVALCANTE5; \\ Jéssica Cristina Barbosa FERREIRA ${ }^{6}$; José Eduardo Macedo PEZZOPANE²
}

\begin{abstract}
RESUMO - A utilização de substratos oriundos de resíduos orgânicos torna-se uma estratégia para produção de mudas, tendo em vista o barateamento do processo de produção e destinação dos resíduos diminuindo eventuais impactos. Nesse sentido, objetivou-se avaliar o potencial dos diferentes resíduos orgânicos regionais como substratos para produção de mudas, utilizando como planta teste a Leucaena leucocephala (Lam.) de Wit. por ser uma espécie rústica e de fácil desenvolvimento. O experimento foi conduzido em uma casa de vegetação com $50 \%$ de luminosidade. Adotou-se o delineamento experimental em blocos inteiramente casualizados com quatro tratamentos (paú de buriti, resíduo de carnaúba decomposta + solo + esterco bovino, resíduo de carnaúba decomposta + casca de arroz e resíduo de carnaúba decomposta + solo) e cinco blocos ou repetições. Cada bloco contou com dez plântulas, totalizando 200 plântulas. Os parâmetros avaliados foram porcentagem de emergência, índice de velocidade de emergência, altura da parte aérea, diâmetro do colo, número de folhas definitivas, comprimento da raiz e matéria seca do sistema radicular. As mudas produzidas nos substratos paú de buriti e resíduo de carnaúba decomposta + solo + esterco bovino emergiram mais (maiores porcentagens de emergência) e apresentaram crescimento inicial maior (maiores alturas da parte aérea), o que leva a inferir que estes substratos propiciaram melhores condições para produção de mudas de L. leucocephala. Fazem-se necessários mais estudos com esses substratos para a produção de mudas, a fim de ampliar seu potencial de utilização.
\end{abstract}

Palavras-chaves: leucena; mudas; diferentes substratos; resíduos orgânicos.

\footnotetext{
${ }^{1}$ Recebido para análise em 22.09.2016. Aceito para publicação em 11.09.2017.

${ }^{2}$ Universidade Federal Espírito Santo - UFES, Programa de Pós-Graduação em Ciências Florestais, Av. Gov. Lindemberg, 316 - Centro, 29550-000, Jerônimo Monteiro, ES, Brasil.

${ }^{3}$ Universidade Federal de Viçosa - UFV, Programa de Pós-Graduação em Ciência Florestal, Campus Universitário, 36570-000, Viçosa, MG, Brasil.

${ }^{4}$ Universidade Federal Rural de Pernambuco - UFRP, Programa de Pós-Graduação em Biometria e Estatística Aplicada, Rua Dom Manuel de Medeiros, S/N - C1, 56300-990, Petrolina, DF, Brasil.

${ }^{5}$ Universidade Federal do Vale do São Francisco - UNIVASF, Colegiado de Engenharia Agronômica, Rodovia BR 407 - KM 12 - Lote 543 PSNC, S/N - C1, 56300-990, Petrolina, DF, Brasil.

${ }^{6}$ Universidade de Brasília - UnB, Programa de Pós-Graduação em Ciência Florestal, Campus Universitário Darcy Ribeiro, Asa Norte, Caixa Postal 4357, 70910-900, Brasília, DF, Brasil.

${ }^{7}$ Autor para correspondência: Genilda Canuto Amaral - genildacanuto@gmail.com
} 


\begin{abstract}
The use of substrates from organic residues becomes a strategy for the production of seedlings, in view of the lowering of the production process and the destination of the residues, reducing eventual impacts. In this sense, the objective was to evaluate the potential of the different regional organic residues as substrates for seedling production, using Leucaena leucocephala (Lam.) de Wit. as a test plant by being a rustic species and of easy development. The experiment was conducted in a greenhouse with $50 \%$ luminosity. The experiment was carried out in a completely randomized block design with four treatments (buriti leaf, decomposed carnauba residue + soil + bovine manure, decomposed carnauba residue + rice husk and decomposed carnauba residue + soil) and five blocks or replicates. Each block had ten seedlings, totaling 200 seedlings. The parameters evaluated were emergency percentage, emergence speed index, shoot height, lap diameter, number of definitive leaves, root length and dry matter of the root system. The seedlings produced in the buriti and carnauba residue substrates decomposed + soil + bovine manure emerged more (higher percentages of emergence) and presented higher initial growth (higher shoot height), which leads to infer that these substrates provided better conditions for seedling production of L. leucocephala. Further studies with these substrates are required for the production of seedlings in order to increase their potential for use.
\end{abstract}

Keywords: leucena; seedlings; different substrates; organic waste.

\section{INTRODUÇÃO}

O desenvolvimento de atividades degradadoras do ambiente vem comprometendo significativamente a sustentabilidade ambiental. Neste sentido, o desenvolvimento de estratégias como a revegetação com espécies arbóreas produzidas em substratos à base de resíduos orgânicos regionais constitui uma importante opção, tendo em vista o barateamento do processo de produção de mudas. Assim, a realização de estudos nesta temática é uma ferramenta fundamental por subsidiar e ajudar nas ações necessárias para recuperação e manejo de áreas degradadas (Lima, 2008).

Entre as espécies arbóreas, Leucaena leucocephala (Lam.) de Wit pode ser considerada como de ampla possibilidade de adaptação em áreas degradadas, seja por sua alta versatilidade na produção de madeira, carvão vegetal e forragem, como recuperação de solos degradados (Empresa Brasileira de Pesquisa Agropecuária - EMBRAPA, 2006). Araújo Filho (2007), em estudo com espécies leguminosas, concluiu que a leucena pode ser utilizada em programas de recuperação florestal de áreas degradadas com sucesso.
Além disso, a mesma possui várias habilidades adaptativas, como capacidade de tolerar diferentes condições de clima e solo, crescimento inicial rápido e maturação precoce, além de realizar a fixação biológica de nitrogênio - FBN, contribuindo para melhoria de atributos químicos e biológicos dos solos (Decker, 2008).

No processo de produção de mudas, de forma geral, um dos fatores imprescindíveis a ser considerado é o substrato utilizado, pois é determinante para o êxito no estabelecimento de plantios, uma vez que este exerce influência sobre a emergência de plântulas e a qualidade das mudas (Alexandre et al., 2006). O tipo de substrato usado deve ser adequado às exigências fisiológicas da germinação, tamanho e forma da semente (Brasil, 2009); além de apresentar características adequadas para um bom desenvolvimento da muda. Além disso, segundo Maciel et al. (2000), os substratos exercem influência significativa na arquitetura do sistema radicular e nas associações biológicas com o meio, influenciando o estado nutricional das plantas e a translocação de água no sistema solo-planta-atmosfera. 
AMARAL, G.C. et al. Emergência e crescimento de plântulas de Leucaena leucocephala em diferentes substratos.

$\mathrm{Na}$ escolha de um substrato, é preciso atentar-se a seu custo e sua disponibilidade, assim como garantir que as propriedades químicas e físicas do mesmo atendam às necessidades de crescimento adequado das plantas (Caldeira et al., 2012). Os substratos podem ser constituídos por um único material ou pela combinação de diferentes tipos de materiais, que podem ser produzidos ou adquiridos em empresas especializadas. No mercado, é possível adquirir diversos tipos de substratos prontos para o uso, puros ou em mistura, cada um com características próprias de preço e qualidade (Kratz, 2011).

Contudo, o uso de materiais renováveis na formulação de substratos é de fundamental importância, haja vista a questão ambiental, bem como, o aumento da produção de mudas, que deve seguir padrões de sustentabilidade. Desse modo, faz-se necessário o fornecimento de novos insumos para serem utilizados como substratos, em busca de apresentar novas formulações, por exemplo, o uso de resíduos agroindustriais, de indústrias florestais e urbanas, representando uma alternativa viável, pois grandes volumes destes produtos são gerados, promovendo um problema ambiental se não for apresentado um destino final adequado (Kratz et al., 2013).

Assim, o trabalho foi realizado com objetivo de avaliar diferentes resíduos orgânicos produzidos na região do Piauí como substratos para produção de mudas, utilizando como planta teste Leucaena leucocephala (Lam.) de Wit. por ser uma espécie rústica e de fácil desenvolvimento.

\section{MATERIAL E MÉTODOS}

O experimento foi conduzido no Viveiro Florestal da Universidade Federal do Piaú, localizado no município de Bom Jesus-Piauí, coordenadas geográficas $09^{\circ} 04^{\prime} 28^{\prime \prime} \mathrm{S}, 4^{\circ} 21^{\prime} 31^{\prime \prime} \mathrm{W}$ com altitude média de $277 \mathrm{~m}$, precipitação média entre 900 a $1.200 \mathrm{~mm} / \mathrm{ano}$ e temperatura média de $26,5^{\circ} \mathrm{C}$ (Viana et al., 2002).

O delineamento experimental utilizado foi o de blocos inteiramente casualizados, utilizando-se quatro tratamentos $\left(\mathrm{S}_{1}=\right.$ paú de buriti, originado da decomposição natural do caule da palmeira buriti (Mauritia flexuosa); $\mathrm{S}_{2}=50 \%$ resíduo de carnaúba decomposta (Copernicia prunifera) +
$25 \%$ solo $+25 \%$ esterco bovino, base volumétrica; $\mathrm{S}_{3}=60 \%$ resíduo de carnaúba (C. prunifera) + $40 \%$ casca de arroz (Oriza sativa) in natura e $\mathrm{S}_{4}=50 \%$ resíduo de carnaúba decomposta $(C$. prunifera $)+50 \%$ solo, base volumétrica. Adotou-se cinco repetições, ou seja, cinco blocos de dez plantas, perfazendo um total de 200 mudas. As mesmas foram mantidas em casa de vegetação com $50 \%$ de luminosidade, com uma irrigação manual diária.

Para o desenvolvimento do experimento, utilizou-se a espécie Leucaena leucocephala, cujas sementes foram adquiridas em loja autorizada. Antes da semeadura, foi realizada a quebra de dormência das sementes através do método de escarificação química (imersão em $\mathrm{H}_{2} \mathrm{SO}_{4}$ concentrado por 20 minutos) conforme metodologia proposta por Teles et al. (2000). Foram distribuídas três sementes por recipiente, em profundidade aproximada de 1,5 cm. Foram utilizados sacos de polietileno nas dimensões de $14 \quad$ x $8 \quad \mathrm{~cm} \quad(0,224 \quad$ L) como recipientes. Os sacos de polietileno receberam os diferentes substratos para compor os tratamentos (cada saco recebeu a mesma quantidade de substrato em base volumétrica, sem adensamento do substrato no recipiente). Após emergência das sementes foi realizado o desbaste, deixando apenas uma plântula por recipiente.

Durante os 30 dias, as plântulas foram avaliadas quanto sua emergência, resultando na porcentagem de emergência - PE e índice de velocidade de emergência - IVE.

A porcentagem de emergência foi calculada de acordo com Labouriau e Valadares (1976):

$$
\mathrm{PE}=(\mathrm{N} / \mathrm{A}) \cdot 100
$$

Em que: PE - porcentagem de emergência; $\mathrm{N}$ - número total de plântulas emergidas; $\mathrm{A}$ - número total de sementes colocadas para germinar.

O índice de velocidade de emergência foi calculado pela fórmula proposta por Maguire (1962):

$$
\mathrm{IVE}=\mathrm{E} 1 / \mathrm{N} 1+\mathrm{E} 2 / \mathrm{N} 2+\ldots+\mathrm{En} / \mathrm{Nn}
$$

Em que: IVE - Índice de velocidade de emergência; E1, E2 e En - número de plântulas normais computadas da primeira à última contagem; N1, N2 e $\mathrm{Nn}$ - número de dias após a implantação do teste. 
AMARAL, G.C. et al. Emergência e crescimento de plântulas de Leucaena leucocephala em diferentes substratos.

Após 30 dias da semeadura (final do experimento), foram analisados os seguintes parâmetros de todas as mudas:

- parâmetros não destrutivos: altura da planta $(\mathrm{cm})$ (medida com o auxílio de régua milimetrada), diâmetro do colo (mm) (medido com o auxílio de paquímetro digital), número de folhas definitivas (determinado por meio da contagem das folhas fotossinteticamante ativas), comprimento do sistema radicular $(\mathrm{cm})$ (medido com o auxílio de régua milimetrada).

- parâmetros destrutivos: as mudas foram separadas em sistema radicular e parte aérea, e acondicionadas em sacos de papel tipo Kraft e colocadas para secar em estufa a $65 \pm 3{ }^{\circ} \mathrm{C}$, com circulação forçada de ar, até peso constante a fim de se obter peso da matéria seca do sistema radicular e da parte aérea (g), com auxílio de balança digital com precisão de 0,0001 g (Mark 250A - Capacidade 250 g). Após a obtenção dos pesos de matéria seca do sistema radicular e da parte aérea, obteve-se o peso da matéria seca total (por meio da soma do peso de matéria seca do sistema radicular e da parte aérea) e a razão entre o peso da matéria seca do sistema radicular e o peso da matéria seca da parte aérea.
Os dados foram submetidos à análise de variância pelo teste "F" para diagnóstico de efeito significativo dos tratamentos; as médias comparadas entre si pelo teste de Tukey $(p<0,05)$, exceto a variável peso da matéria seca total que foi submetida ao teste de Duncan ( $\mathrm{p}<0,05$ ), sendo empregado o software ASSISTAT (Silva e Azevedo, 2009) para avaliação de diferença significativa.

\section{RESULTADOS E DISCUSSÃO}

De acordo com a análise de variância da emergência de sementes de L. leucocephala (Tabela 1), verificou-se que os tipos de substratos exerceram efeitos significativos $(\mathrm{p}<0,05)$ apenas para porcentagem de emergência - PE, não influenciando o índice de velocidade de emergência - IVE.

Para a porcentagem de emergência (Figura 1), as sementes semeadas nos substratos $\mathrm{S}_{1}$ (paú de buriti), $\mathrm{S}_{2}$ (50\% resíduo de carnaúba decomposta (C. prunifera) $+25 \%$ solo $+25 \%$ esterco bovino, base volumétrica), $\mathrm{S}_{3}(60 \%$ resíduo de carnaúba (C. prunifera) + casca de arroz (O. sativa) in natura) apresentaram maiores porcentagens de emergência (86, 90 e 78\%, respectivamente) e não se diferenciaram estatisticamente entre si, porém quando semeadas em $\mathrm{S}_{2}$ apresentaram maiores porcentagens $(90 \%)$.

Tabela 1. Análise de variância da porcentagem de emergência e índice de velocidade de emergência de L. leucocephala cultivada em diferentes substratos.

Table 1. Analysis of variance of emergence percentage and rate of emergence of L. leucocephala grown on different substrates.

\begin{tabular}{ccccc}
\hline Parâmetros & QM Tratamento & QM Resíduo & F & CV \\
\hline Porcentagem de emergência & 3900,00 & 2120,00 & $9,81^{*}$ & 14,95 \\
Índice de velocidade de emergência & 55,84 & 102,45 & $2,90 \mathrm{~ns}$ & 30,69 \\
\hline
\end{tabular}

QM: Quadrado médio; CV: coeficiente de variação; ns: não significativo a 5\% de significância; *: significativo ao nível de 5\% de significância pelo teste $\mathrm{F}$.

QM: medium square; CV: coefficient of variance; ns: not significant at 5\% significance; *: significant at a level of $5 \%$ of significance by test $\mathrm{F}$. 


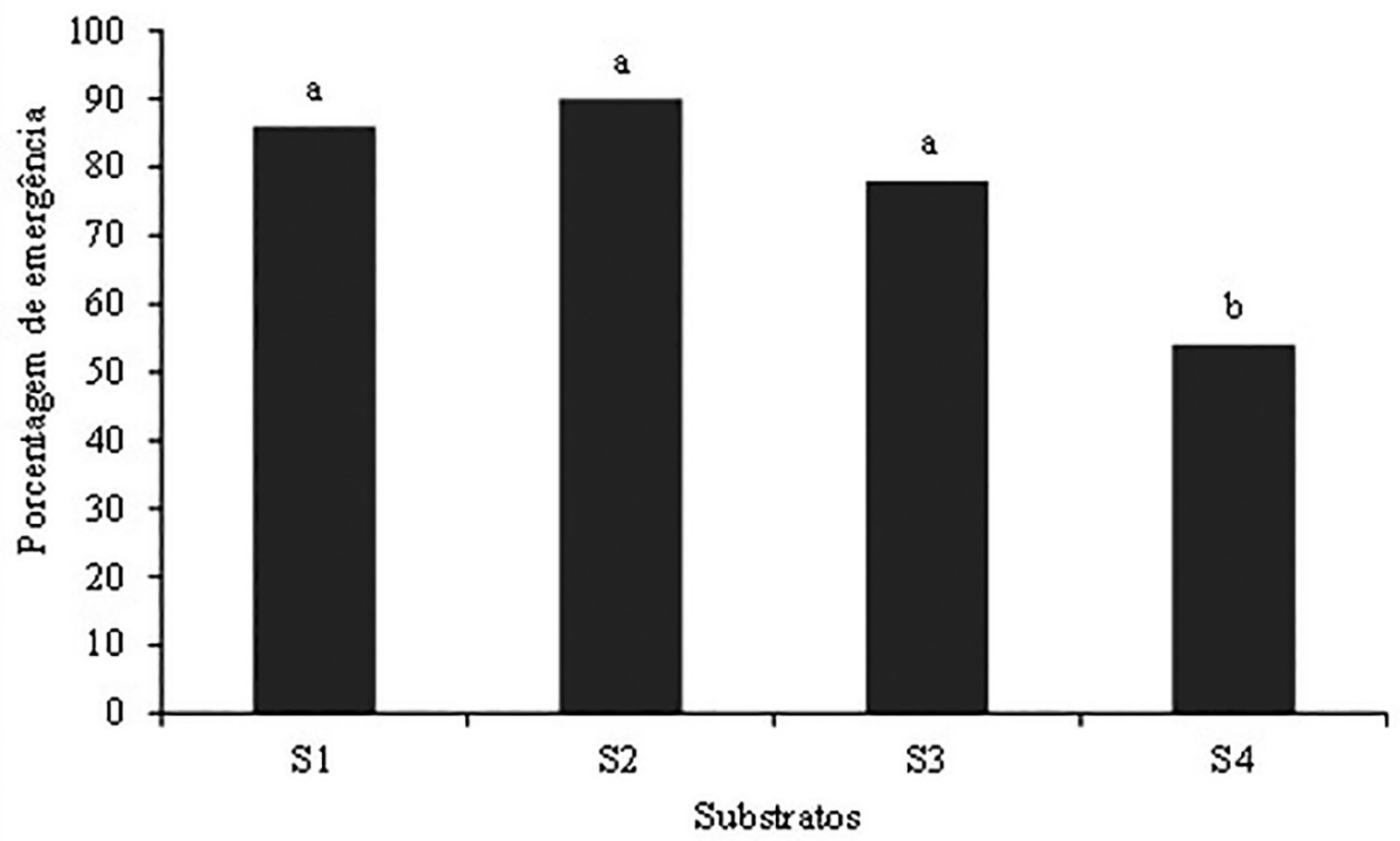

Figura 1. Porcentagem de emergência de L. leucocephala em diferentes substratos: $\mathrm{S}_{1}=$ paú de buriti; $\mathrm{S}_{2}=50 \%$ resíduo de carnaúba decomposta $(C$. prunifera $)+25 \%$ solo $+25 \%$ esterco bovino, base volumétrica; $\mathrm{S}_{3}=60 \%$ resíduo de carnaúba (C. prunifera) $+40 \%$ casca de arroz $\left(O\right.$. sativa) in natura e $\mathrm{S}_{4}=50 \%$ resíduo de carnaúba decomposta (C. prunifera $)+50 \%$ solo, base volumétrica.

Figure 1. L. leucocephala percentage of emergency on different substrates: $\mathrm{S}_{1}=$ paú de buriti; $\mathrm{S}_{2}=60 \%$ decomposed carnauba residue $(C$. prunifera $)+25 \%$ soil $+25 \%$ bovine manure, volumetric base; $\mathrm{S}_{3}=60 \%$ carnauba residue $(C$. prunifera $)+40 \%$ rice husks $(O$. sativa $)$ in natura and $\mathrm{S}_{4}=50 \%$ decomposed carnauba residue (C. prunifera $)+50 \%$ soil, volumetric base.

Quando semeadas no substrato $\mathrm{S}_{4}$ (50\% resíduo de carnaúba decomposta (C. prunifera) $+50 \%$ solo, base volumétrica), apresentaram menores porcentagens de emergência (54\%). Cabe ressaltar que as sementes germinam quando são oferecidas condições favoráveis de temperatura e umidade, daí a importância de se obter um substrato que proporcione o conjunto de características adequadas à germinação das mesmas, uma vez que o substrato é caracterizado como um composto adequado para a sustentação e retenção de quantidades suficientes e necessárias de água, oxigênio e nutrientes, além de oferecer $\mathrm{pH}$ compatível, ausência de elementos químicos em teores tóxicos e condutividade elétrica adequada (Guerrini e Trigueira, 2004).
A germinação rápida e uniforme, seguida por uma imediata emergência das plântulas, são características altamente desejáveis, pois quanto mais tempo a plântula permanecer nos estágios iniciais de desenvolvimento e demorar a emergir no solo, mais vulnerável estará às condições adversas do meio (Martins et al., 1999).

Para o crescimento inicial das mudas de leucena (Tabela 2), nas condições do experimento, verificou-se que os substratos testados exerceram efeitos significativos $(\mathrm{p}<0,05)$ na altura das plantas, peso da matéria seca do sistema radicular e peso da matéria seca total; contudo, para número de folhas, diâmetro do colo, comprimento da raiz, peso da matéria seca da parte aérea e razão do peso da matéria seca da parte aérea pelo peso da matéria seca do sistema radicular, esse efeito não foi observado. 
AMARAL, G.C. et al. Emergência e crescimento de plântulas de Leucaena leucocephala em diferentes substratos.

Tabela 2. Análise de variância do crescimento inicial em termos de altura da muda, número de folhas, diâmetro do colo, comprimento do sistema radicular, peso da matéria seca do sistema radicular, peso da matéria seca da parte área, peso da matéria seca total e relação do peso da matéria seca da parte aérea pelo peso da matéria seca do sistema radicular de mudas de L. leucocephala cultivadas em diferentes substratos, 30 dias após a semeadura.

Table 2. Analysis of variance of the initial growth in terms of seed height, number of leaves, neck diameter, root system length, dry matter weight of the root system, dry matter weight of the aereal part, total dry matter weight and weight of the dry matter of the aerial part by the weight of the dry matter of the root system of L. leucocephala seedlings grown on different substrates, 30 days after sowing.

\begin{tabular}{lcccc}
\hline \multicolumn{1}{c}{ Parâmetros } & QM Tratamento & QM Resíduo & F & CV \\
\hline Altura de planta & 38,64 & 36,91 & $5,58^{*}$ & 14,22 \\
Diâmetro do colo & 0,35 & 1,11 & $1,71 \mathrm{~ns}$ & 12,83 \\
Número de folhas & 21,31 & 60,83 & $1,86 \mathrm{~ns}$ & 16,89 \\
Comprimento do sistema radicular & 32,68 & 86,90 & $2,00 \mathrm{~ns}$ & 10,47 \\
Peso da matéria seca sistema radicular - PMSSR & 0,51 & 0,76 & $3,57^{*}$ & 28,78 \\
Peso da matéria seca - parte aérea - PMSPA & 3,39 & 6,13 & $2,95 \mathrm{~ns}$ & 38,38 \\
Peso da matéria seca total & 6,26 & 9,60 & $3,47^{*}$ & 32,62 \\
PMSPA/PMSSR & 1,26 & 6,35 & $1,06 \mathrm{~ns}$ & 29,53 \\
\hline
\end{tabular}

QM: Quadrado médio; CV: coeficiente de variação (\%); ns: não significativo a 5\% de significância; *: significativo ao nível de 5\% de significância pelo teste $\mathrm{F}$.

QM: Medium square; CV: coefficient of variance; ns: not significant at 5\% significance; *: significant at a level of $5 \%$ of significance by test $\mathrm{F}$.

Analisando os valores médios da altura das mudas de L. leucocephala (Figura 2), nota-se que as mudas conduzidas em substrato $\mathrm{S}_{2} \quad(50 \%$ resíduo de carnaúba decomposta $($ C. prunifera $)+25 \%$ solo $+25 \%$ esterco bovino, base volumétrica) e $\mathrm{S}_{1}$ (paú de buriti) cresceram mais quando comparadas às mudas conduzidas nos outros substratos testados, 12,42 e 11,22 cm, respectivamente, embora não tenha havido diferença estatística em relação às mudas conduzidas em $\mathrm{S}_{3} \quad(60 \%$ resíduo de carnaúba (C. prunifera) $+40 \%$ casca de arroz) com $10,49 \mathrm{~cm}$. Provavelmente, a presença da matéria orgânica na composição do substrato $\left(\mathrm{S}_{2}\right)$, fornecido pelo esterco bovino, influenciou positivamente no crescimento das mudas. Por outro lado, o desenvolvimento das mudas conduzidas em substrato $\mathrm{S}_{4}(50 \%$ resíduo de carnaúba decomposta (C. prunifera) $+50 \%$ solo, base volumétrica) foi menor quando comparado ao desenvolvimento das mudas produzidas nos outros substratos (altura de 8,58 cm). Lucena et al. (2006), trabalhando com a mesma espécie, observaram, aos 180 dias após a semeadura, menores alturas $(13,2 \mathrm{~cm})$ nas mudas desenvolvidas em solo utilizado como substrato e maiores alturas $(41,3 \mathrm{~cm})$ ao utilizar o solo + esterco bovino (1:1).
Silva Junior et al. (2014), analisando as características físicas e químicas do substrato paú de buriti, resíduo da carnaúba semidecomposto e resíduo da carnaúba + casca de arroz, observaram boas características dos mesmos ( $\mathrm{N}, \mathrm{P}, \mathrm{K}, \mathrm{Ca}, \mathrm{Mg}$ e capacidade de retenção de água, espaço de aeração, respectivamente), podendo desempenhar bons resultados como substrato para produção de mudas.

Substratos que contêm maior percentual de matéria orgânica apresentam boa capacidade de retenção de água e aeração, além da quantidade de nutrientes disponíveis para a planta (Araújo Neto et al., 2002).

A altura e o diâmetro do coleto estão entre os mais importantes parâmetros morfológicos para estimar o crescimento de mudas (Carneiro, 1995). Além disso, na fase de campo, os parâmetros acima são importantes para uma boa estabilidade e desenvolvimento das mudas. Oliveira et al. (2010), trabalhando com leucena, encontraram, aos 330 dias, resultados satisfatórios para altura $(1,46 \mathrm{~m})$ e diâmetro de coleto $(9,02 \mathrm{~mm})$ das mudas. 


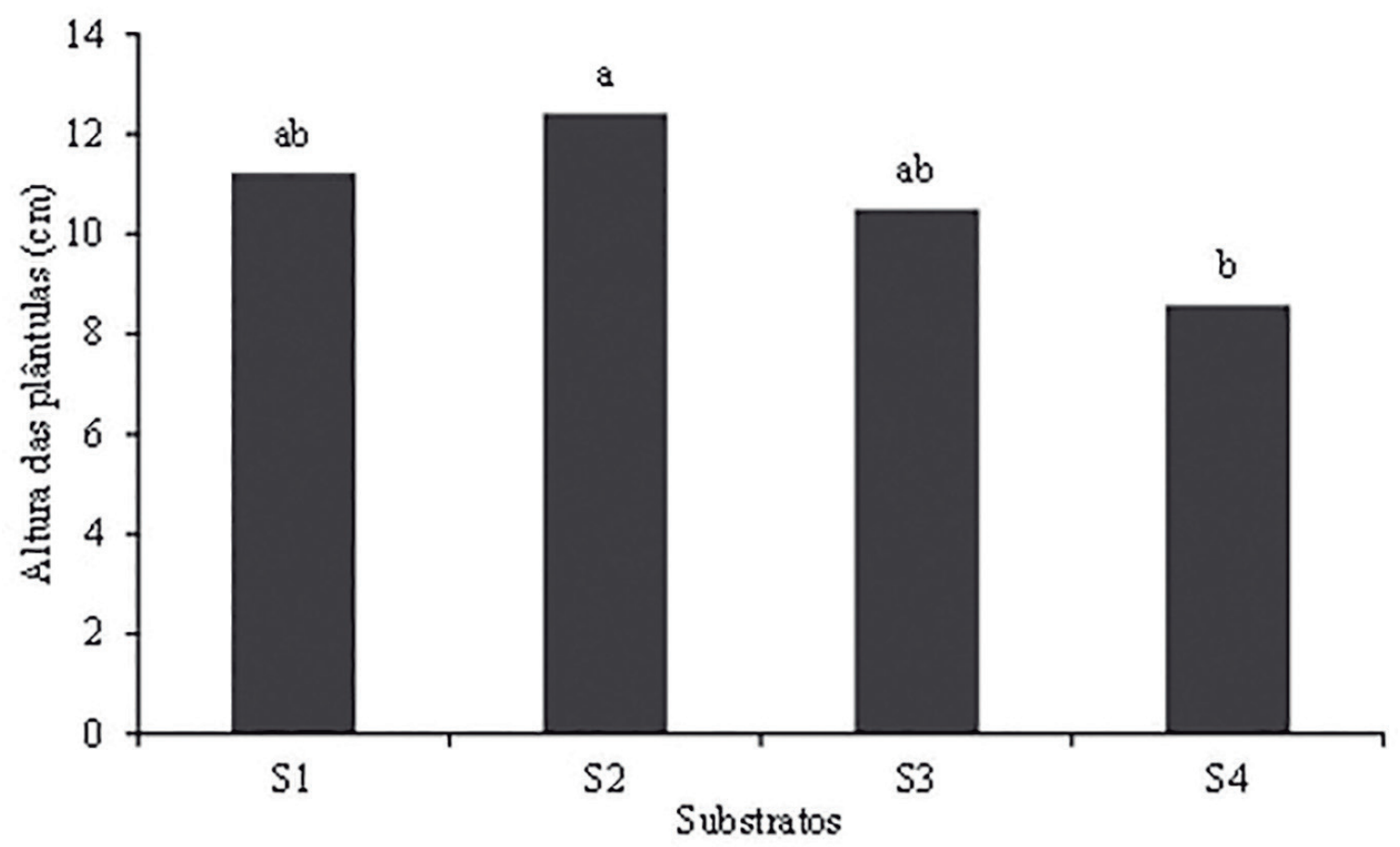

Figura 2. Alturas médias de plântulas de L. leucocephala produzidas em diferentes substratos: $\mathrm{S}_{1}=$ paú de buriti; $\mathrm{S}_{2}=50 \%$ resíduo de carnaúba decomposta $(C$. prunifera $)+25 \%$ solo $+25 \%$ esterco bovino, base volumétrica; $\mathrm{S}_{3}=60 \%$ resíduo de carnaúba (C. prunifera) $+40 \%$ casca de arroz (O. sativa) in natura e $\mathrm{S}_{4}=50 \%$ resíduo de carnaúba decomposta (C. prunifera) $+50 \%$ solo, base volumétrica.

Figure 2. Average heights of $L$. leucocephala seedlings produced on different substrates: $\mathrm{S}_{1}=$ paú de buriti; $\mathrm{S}_{2}=60 \%$ decomposed carnauba residue $(C$. prunifera $)+25 \%$ soil $+25 \%$ bovine manure, volumetric base; $\mathrm{S}_{3}=60 \%$ carnauba residue $(C$. prunifera $)+40 \%$ rice husks $\left(O\right.$. sativa) in natura and $\mathrm{S}_{4}=50 \%$ decomposed carnauba residue (C. prunifera) $+50 \%$ soil, volumetric base.

Com relação ao peso de matéria seca do sistema radicular (Figura 3), houve diferença estatística entre os tratamentos, sendo que as mudas conduzidas no substrato $\mathrm{S}_{2}(50 \%$ resíduo de carnaúba decomposta (C. prunifera) $+25 \%$ solo $+25 \%$ esterco bovino, base volumétrica) apresentaram, em média, os maiores valores (0,98 g.plântula $\left.{ }^{-1}\right)$, apesar de não terem promovido diferenças estatísticas em relação às médias obtidas para as mudas produzidas em substrato $\mathrm{S}_{1}$ (paú de buriti, originado da decomposição natural do caule da palmeira Mauritia flexuosa) com 0,82 g.planta-1 e $\mathrm{S}_{3}(60 \%$ de resíduo de carnaúba (C. prunifera) + casca de arroz (O. sativa) in natura) com 0,69 g.plântula ${ }^{-1}$. Os menores valores para esse parâmetro foram obtidos nas mudas produzidas no substrato $\mathrm{S}_{4}(50 \%$ resíduo de carnaúba decomposta (C. prunifera $)+50 \%$ solo, base volumétrica) com
0,54 g.planta-1 . Acredita-se que as raízes tenham se desenvolvido mais no substrato constituído por resíduo de carnaúba com casca de arroz em função de suas características físico-químicas (capacidade de retenção de água, espaço de aeração, volume de poros e, condutividade elétrica, N, P, K e $\mathrm{Ca}$ e $\mathrm{Mg}$, respectivamente) resultado encontrado por Silva Junior et al. (2014), pois como afirmado por Freitas et al. (2005), o suprimento de água e nutrientes, a resistência mecânica, a aeração do solo e as interações entre esses fatores influenciam o crescimento de raízes e suas funções. Iossi et al. (2003), ao testarem diferentes substratos, verificaram que a areia e a vermiculita possibilitaram maior desenvolvimento da raiz primária de plântulas de Phoenix oebelenii. 


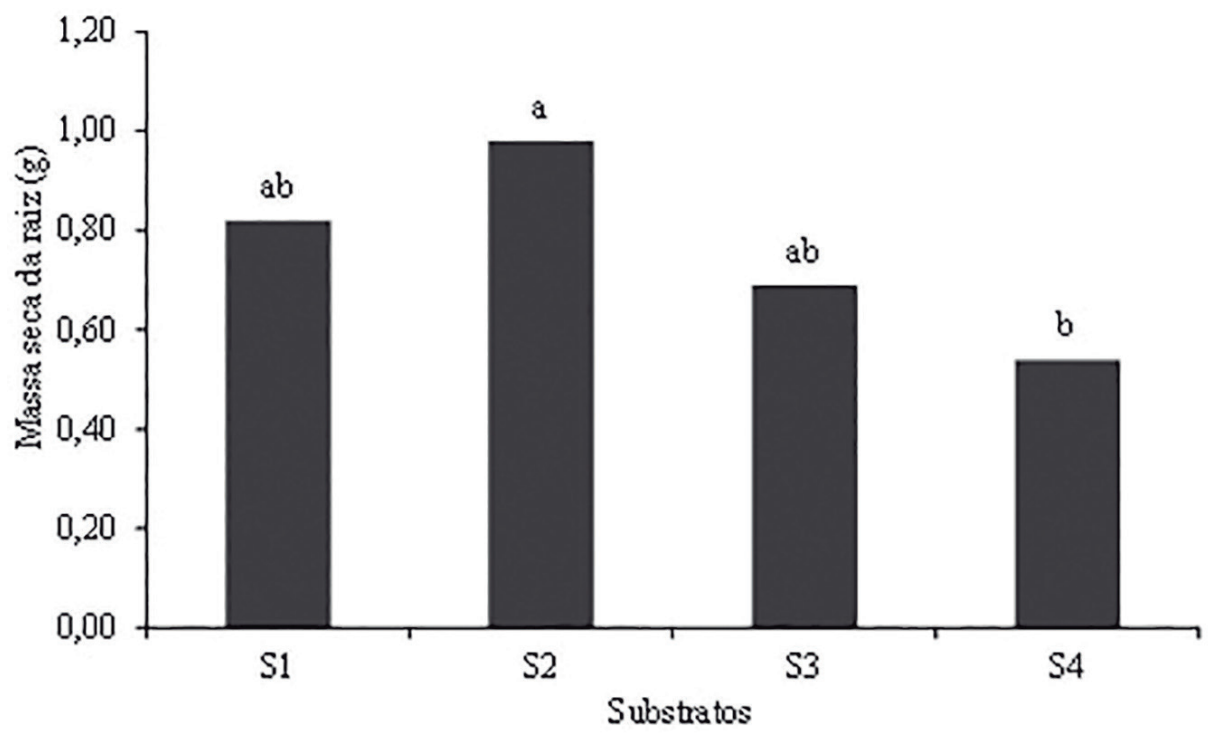

Figura 3. Peso da matéria seca do sistema radicular de mudas de L. leucocephala conduzidas em diferentes substratos: $\mathrm{S}_{1}=$ paú de buriti; $\mathrm{S}_{2}=50 \%$ resíduo de carnaúba decomposta (C. prunifera) $+25 \%$ solo $+25 \%$ esterco bovino, base volumétrica; $\mathrm{S}_{3}=60 \%$ resíduo de carnaúba $+40 \%$ casca de arroz (O. sativa) in natura e $\mathrm{S}_{4}=50 \%$ resíduo de carnaúba decomposta (C. prunifera) $+50 \%$ solo, base volumétrica.

Figure 3. Dry matter weight of root system of L. leucocephala seedlings conducted on different substrates: $\mathrm{S}_{1}=$ paú de buriti; $\mathrm{S}_{2}=60 \%$ decomposed carnauba residue $(C$. prunifera $)+25 \%$ soil $+25 \%$ bovine manure, volumetric base; $\mathrm{S}_{3}=60 \%$ carnauba residue $(C$. prunifera $)+40 \%$ rice husks $(O$. sativa $)$ in natura and $\mathrm{S}_{4}=50 \%$ decomposed carnauba residue (C. prunifera) $+50 \%$ soil, volumetric base.

Quanto à produção de matéria seca total, nota-se que ocorreu uma diferença estatística entre os tratamentos (Figura 4). As mudas produzidas nos substratos $\mathrm{S}_{1}$ (paú de buriti, originado da decomposição natural do caule da palmeira (M. flexuosa) e $\mathrm{S}_{2}(50 \%$ resíduo de carnaúba decomposta $(C$. prunifera $)+25 \%$ solo $+25 \%$ esterco bovino, base volumétrica) apresentaram os maiores valores deste parâmetro (ambos 2,90 g), em comparação às mudas conduzidas nos outros substratos testados. Por outro lado, as mudas produzidas em substrato $\mathrm{S}_{4} \quad(50 \%$ resíduo de carnaúba decomposta (C. prunifera) $+50 \%$ solo, base volumétrica) apresentaram menores médias $(1,57 \mathrm{~g})$ de peso de matéria seca total. O maior acúmulo de matéria seca das mudas confere maior suporte ao seu desenvolvimento inicial em campo, características fornecidas pelos substratos $\mathrm{S}_{1}$ e $\mathrm{S}_{2}$. Entre as partes da planta, o caule contribui significativamente para o peso de matéria seca da parte aérea, e quando bem desenvolvido confere uma melhor resistência aos períodos de déficit hídrico no campo, devido ao acúmulo de reservas
(Lima et al., 2006). Para Gomes e Paiva (2004), o peso de matéria seca é um bom indicativo da capacidade de resistência das mudas nas condições de campo.

Amaral et al. (2016) afirmam que o resíduo de carnaúba decomposta apresenta potencial na composição de substratos para produção de mudas de leucena. Além disso, segundo Silva Júnior et al. (2014), a utilização do paú de buriti, material originado da decomposição natural do caule da palmeira buriti (M. flexuosa) e bagana de carnaúba (C. prunifera) - resíduo das fábricas que extraem o pó cerífero das folhas da carnaúba na região do Nordeste brasileiro - é uma boa oportunidade para desenvolver materiais regionais com potencial. A aquisição desses substratos não caracteriza exploração da palmeira de buriti e muito menos da carnaúba, tendo em vista que o paú de buriti é obtido da palmeira já em estado de decomposição natural e o substrato a base de carnaúba é adquirido de resíduos. A destinação desses materiais como potenciais substratos na produção de mudas consiste no reaproveitamento dos resíduos. 


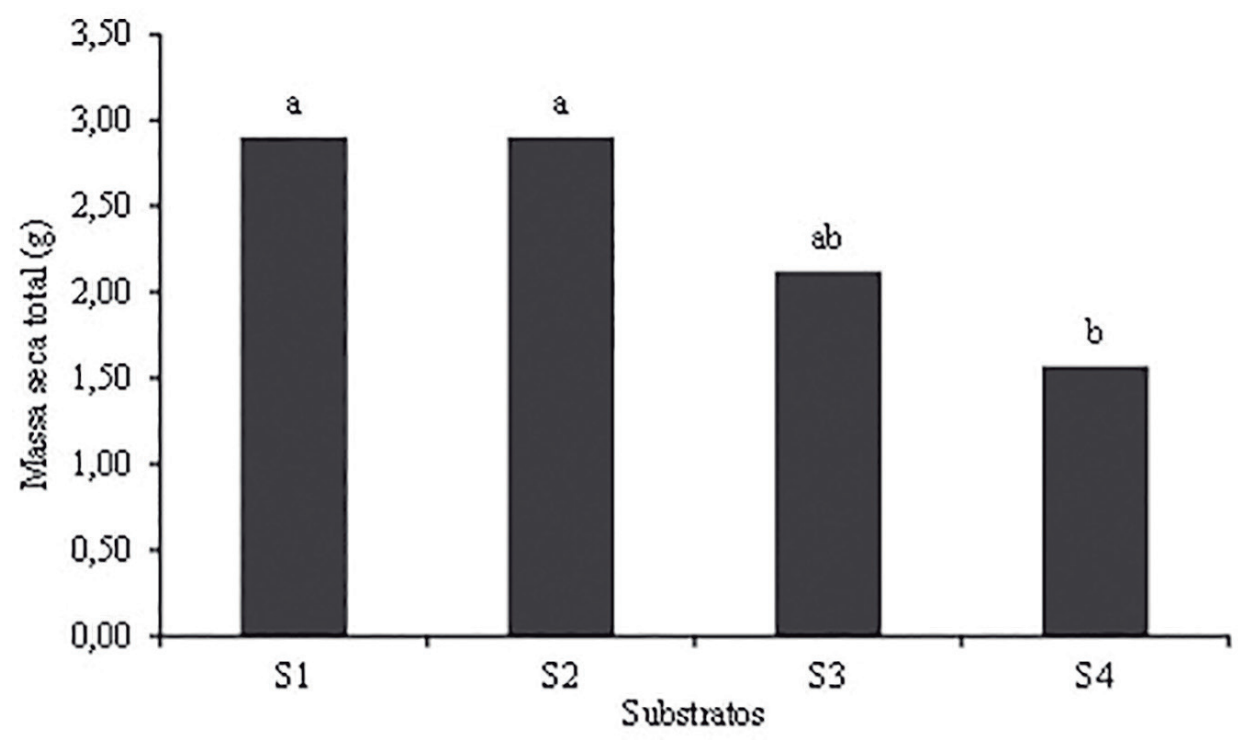

Figura 4. Peso da matéria seca total de mudas de L. leucocephala produzidas em diferentes substratos: $\mathrm{S}_{1}=$ paú de buriti; $\mathrm{S}_{2}=50 \%$ resíduo de carnaúba decomposta (C. prunifera) $+25 \%$ solo $+25 \%$ esterco bovino, base volumétrica; $\mathrm{S}_{3}=60 \%$ resíduo de carnaúba $+40 \%$ casca de arroz $\left(O\right.$. sativa) in natura e $\mathrm{S}_{4}=50 \%$ resíduo de carnaúba decomposta (C. prunifera $)+50 \%$ solo, base volumétrica.

Figure 4. Total dry matter weight of L. leucocephala seedlings produced on different substrates: $\mathrm{S}_{1}=$ paú de buriti; $\mathrm{S}_{2}=60 \%$ decomposed carnauba residue $(C$. prunifera $)+25 \%$ soil $+25 \%$ bovine manure, volumetric base; $\mathrm{S}_{3}=60 \%$ carnauba residue (C. prunifera) $+40 \%$ rice husks $\left(O\right.$. sativa) in natura and $\mathrm{S}_{4}=50 \%$ decomposed carnauba residue (C. prunifera) $+50 \%$ soil, volumetric base.

Oliveira et al. (2010), analisando a sobrevivência da leucena em campo, obtiveram resultados satisfatórios com $91,6 \%$ de sobrevivência das mudas avaliadas por um período entre 30 e 330 dias após o plantio. Esses resultados também foram encontrados por Shelton (2001) avaliando a sobrevivência, os autores afirmam ainda que a leucena é resistente a seca e de fácil adaptação a solos de baixa fertilidade.

A leucena é uma espécie exótica com potencial para recuperação de áreas degradadas por apresentar características da adaptabilidade a diferentes condições de solo e clima, desenvolvimento rápido, além de fixação de nitrogênio, contribuindo para os atributos químicos e biológicos do solo (Decker, 2008). Por apresentar tais características, necessita de cuidados ao planejar a recuperação de uma área com introdução de tal espécie, pois por ser exótica não deve ser dominante na área, visto que pode se tornar um problema futuro na área. Sua utilização se justifica principalmente pelos atributos citados, pois promove a recuperação das características químicas e físicas do solo.

\section{CONCLUSÕES}

A emergência e o crescimento inicial de plântulas de L. leucocephala são positivamente influenciados ao se fazer uso de substratos que contenham matéria orgânica em sua composição.

Os substratos paú de buriti e resíduo de carnaúba decomposta + solo + esterco bovino são materiais potenciais para produção de mudas de L. leucocephala.

\section{REFERÊNCIAS BIBLIOGRÁFICAS}

ALEXANDRE, R.S. et al. Estádio de maturação dos frutos e substratos na germinação de sementes e desenvolvimento inicial de plântulas de jabuticabeira. Revista Brasileira de Agrociência, v. 12, n. 2, p. 227-230, 2006. 
AMARAL, G.C. et al. Emergência e crescimento de plântulas de Leucaena leucocephala em diferentes substratos.

AMARAL, F.H.C. et al. Growth of Leucaena leucocephala (Lam.) de Wit favored by organic waste in the Brazilian semiarid region. Revista Brasileira de Engenharia Agrícola e Ambiental, v. 20, n. 7, p. 612-617, 2016.

ARAÚJO FILHO, J.A. de. Avaliação de leguminosas arbóreas, para recuperação de solos e repovoamento em áreas degradadas, Quixeramobim - CE. Revista Brasileira de Agroecologia, v. 2, n. 2, 2007.

ARAÚJO NETO, S.E. et al. Desenvolvimento de mudas de maracujazeiro amarelo com uso de diferentes substratos e recipientes. In: CONGRESSO BRASILEIRO DE FRUTICULTURA, 17., 2002, Belém. Anais... Belém: SBF. 1 CD-ROM.

BRASIL. Ministério da Agricultura, Pecuária e Abastecimento. Regras para análise de sementes. Brasília, DF: MAPA/ACS, 2009. 365 p.

CALDEIRA, M.V.W. et al. Biossólido na composição de substrato para a produção de mudas de Tectona grandis. Revista Floresta, v. 23, n. 12, p. 77-84, 2012.

DECKER, V. Avaliação da intensidade luminosa na germinação e no desenvolvimento de leucena (Leucaena leucocephala (Lam.) de Wit). 2008. 65 f. Dissertação (Mestrado em Agronomia) - Universidade Estadual do Oeste do Estado do Paraná - UNIOESTE, Campus Marechal Cândido Rondon, Marechal Cândido Rondon.

EMPRESA BRASILEIRA DE PESQUISA AGROPECUÁRIA - EMBRAPA. Leucena (Leucaena spp.) legumineira - cultura forrageira para produção de proteína. São Paulo: Embrapa Gado de Corte, 2006.

FREITAS, T.A.S. et al. Desempenho radicular de mudas de eucalipto produzidas em diferentes recipientes e substratos. Revista Árvore, v. 29, n. 6, p. 853-861, 2005.
GOMES, J.M.; PAIVA, H.N. Viveiros florestais propagação sexuada. 3. ed. Viçosa: UFV, 2004. $116 \mathrm{p}$.

GUERRNI, I.A.; TRIGUEIRA, R.M. Atributos físicos e Químicos de Substratos Compostos por Biossólos e Casca de Arroz Carbonizada. Revista Brasileira Ciência do Solo, v. 28, p. 1069-1076, 2004.

IOSSI, E. et al. Efeitos de substratos e temperaturas na germinação de sementes de tamareira-anã (Phoenix roebelenii O'Brien). Revista Brasileira de Sementes, v. 25, n. 2, p. 63-69, 2003.

KRATZ, D. Substratos renováveis na produção de mudas de Eucalyptus benthamii Maiden et Cambage e Mimosa scabrella Benth. 2011. 121 f. Dissertação (Mestrado em EngenhariaFlorestal) - Universidade Federal do Paraná, Curitiba.

.; WENDLING, A.C.N.; SOUZA, P.V.D. Substratos renováveis na produção de mudas de Eucalyptus benthamii. Ciência Florestal, v. 23, n. 4, p. 607-621, 2013.

LABORIAU, L.G.; VALADARES, M.B. On the germination of seeds of Calotropis procera. Anais da Academia Brasileira de Ciências, São Paulo, v.48, p.174-186. 1976.

LIMA, E.P.C. Florística e estrutura de uma área degradada por garimpo de ouro abandonado e do remanescente florestal de entorno, no município de Alta Floresta/MT. 2008. 72 f. Dissertação (Especialização em Ciências Ambientais) - Departamento de Ciências Ambientais, Universidade do Estado do Mato Grosso, Cáceres.

LIMA, J.D.; SILVA, B.M.S.; MORAES, W.S. Efeito da luz no crescimento de plântulas de Virola surinamensis (Rol.) Warb. Revista Científica Eletrônica de Engenharia Florestal, v. 4, n. 8, 2006. 
AMARAL, G.C. et al. Emergência e crescimento de plântulas de Leucaena leucocephala em diferentes substratos.

LUCENA, A.M.A; GUERRA, H.O.C; CHAVES, L.H.G. Desenvolvimento de mudas de leucena e flamboyant em diferentes composições de substratos. Revista Verde, v. 1, n. 2, p. 16-23, 2006.

MACIEL, A.L.R.; SILVA, A.B.; PASQUAL, M. Aclimatação de plantas de violeta (saintpaulia ionantha wendl) obtidas in vitro: efeitos do substrato. Ciência e Agrotecnologia, v. 24, n. 1, p. 9-12, 2000 .

MAGUIRE, J.D. Speeds of germination-aid selection and evaluation for seedling emergence and vigor. Crop Science, v. 2, p. 176-177, 1962.

MARTINS, C.C.; NAKAGAWA, J.; BOVI, M.L.A. Efeito da posição da semente no substrato e no crescimento inicial das plântulas de palmito-vermelho (Euterpe espiritosantensis Fernades - Palmae). Revista Brasileira de Sementes, v. 21, p. 164-173, 1999.

OLIVEIRA, P.D. et al. Estabelecimento de sistema silvipastoril com leucena em unidade de produção familiar no sudeste do Pará. Enciclopédia Biosfera, Centro Científico Conhecer, v. 6, n. 11, p. 1-7, 2010.

SHELTON, H.M. Potenciais e limitações de Leucaena spp. para uso em sistemas silvipastoris. In: CARVALHO, M.M.; ALVIM, M.J.; CARNEIRO, J.C. (Ed.). Sistemas agroflorestais pecuários: opções de sustentabilidade para áreas tropicais e subtropicais. Juiz de Fora: Embrapa Gado de Leite; Brasília, DF: FAO, 2001. p. 379-398.

SILVA, F.A.S.; AZEVEDO, C.A.V. Principal components analysis in the software AssistatStatistical Attendance. In: WORLD CONGRESS ON COMPUTERS IN AGRICULTURE, 7., 2009, Orlando. Proceedings... Reno: American Society of Agricultural and Biological Engineers, 2009. 1 CD-ROM.
SILVA JÚNIOR, J.V. et al. Aproveitamento de materiais alternativos na produção de mudas de tomateiro sob adubação foliar. Revista Ciência Agronômica, v. 45, n. 3, p. 528-536, 2014.

TELES, M.M. et al. Métodos para quebra da dormência em sementes de leucena (Leucaena leucocephala (Lam.) de Wit. Revista Brasileira de Zootecnia, v. 29, n. 2, p. 387-391, 2000.

VIANA, T.V.A. et al. Estudo da aptidão agroclimática do Estado do Piauí para o cultivo da aceroleira. Ciência Agronômica, v. 33, n. 2, p. 5-12, 2002. 